\section{PREVALÊNCIA DE TUBERCULOSE PULMONAR E SINTOMÁTICOS RESPIRATÓRIOS EM UM HOSPITAL PSIQUIÁTRICO DE REFERÊNCIA EM ALAGOAS}

\author{
Prevalence of pulmonary tuberculosis and respiratory \\ symptomatic subjects in a referral psychiatric hospital in \\ Alagoas State
}
Prevalencia de tuberculosis pulmonar y sintomáticos respiratorios de un hospital psiquiátrico de referencia de Alagoas

\section{RESUMO}

Objetivo: Avaliar a prevalência de tuberculose pulmonar (TBP) bacilífera e sintomáticos respiratórios (SR) em um hospital psiquiátrico público de referência em Alagoas, Brasil. Métodos: Pesquisa transversal quantitativa conduzida nas alas de internação do Hospital Escola Portugal Ramalho por meio de questionários contendo dados demográficos, epidemiológicos e sociais, de março a abril de 2013. Grupos estabelecidos: SR, submetidos às coletas para baciloscopia, e assintomáticos (AR). Resultados: Participaram do estudo 143 pacientes, sendo $61,5 \%(n=88)$ homens, 51,7\% $(n=74)$ adultos jovens, 30,8\% $(n=44)$ analfabetos e 41,3\% (n=59) com alguma condição debilitante associada. A prevalência de TBP bacilífera encontrada foi de $0,69 \%(n=1)$. Os SR representaram $14,7 \%(n=21)$ da amostra e foram associados ao grupo de pacientes internados há mais de dois anos e sem perspectiva de alta $(\mathrm{p}=0,01)$, e ao hábito de fumar $(\mathrm{p}=0,02)$. Conclusão: Encontrou-se nesse grupo uma alta prevalência de TBP bacilífera, se comparada à da população brasileira, sendo os SR associados ao maior tempo de internação hospitalar e ao hábito de fumar.

Descritores: Tuberculose Pulmonar; Hospital Psiquiátrico; Hábito de Fumar.

\section{ABSTRACT}

Objective: To evaluate the prevalence of bacilliferous pulmonary tuberculosis (PTB) and respiratory symptomatic $(R S)$ subjects in a referral public psychiatric hospital in Alagoas, Brazil. Methods: Quantitative cross-sectional research conducted in the hospital wards of Portugal Ramalho Hospital School by means of questionnaire forms addressing demographic, epidemiological and social data, from March to April 2013. Groups established: RS, which underwent sputum collections for bacilloscopy, and asymptomatic subjects (RA). Results: The study included 143 participants, with $61.5 \%(n=88)$ males, $51.7 \%(n=74)$ young adults, $30.8 \%(n=44)$ illiterate and $41.3 \%(n=59)$ presenting some debilitating comorbid condition. The prevalence of bacilliferous PTB was found at $0.69 \%(n=1)$. RS represented $14.7 \%$ $(n=21)$ of the sample and were associated with the group of patients hospitalized for over two years and without prospect of discharge $(p=0.01)$, and to smoking $(p=0.02)$. Conclusion: A high prevalence of bacilliferous PTB was found in this group, compared to that found in the Brazilian population, and RS subjects were associated with longer hospitalization length and smoking.

Descriptors: Pulmonary Tuberculosis; Psychiatric Hospitals; Smoking.
Artigo Original

George Vasconcelos Calheiros de Oliveira Costa ${ }^{(1)}$

Larice Caroline de Barros Silva ${ }^{(1)}$ Jairo Calado Cavalcante ${ }^{(1)}$ Valfrido Leão de Melo Neto ${ }^{(1)}$ Sandra Reis Duarte ${ }^{(1)}$

1) Universidade Federal de Alagoas - UFAL - Maceió (AL) - Brasil

Recebido em: 12/07/2015 Revisado em: 10/10/2015 Aceito em: 07/11/2015 


\section{RESUMEN}

Objetivo: Evaluar la prevalencia de la tuberculosis pulmonar (TBP) bacilifera y de los sintomáticos respiratorios de un hospital público psiquiátrico de referencia en Alagoas, Brasil. Métodos: Investigación transversal cuantitativa realizada en las alas de internación del Hospital Escuela Portugal Ramalho a través de cuestionarios con datos demográficos, epidemiológicos y sociales entre marzo y abril de 2013. Grupos establecidos: los Sintomáticos Respiratorios (SR), conducidos a realizar la baciloscopia y los asintomáticos respiratorios (AR). Resultados: Participaron del estudio 143 pacientes, el 61,5\% $(n=88)$ hombres, el 51,7\% $(n=74)$ adultos jóvenes, el 30,8\% $(n=44)$ analfabetos y el 41,3\% $(n=59)$ con alguna debilidad asociada. La prevalencia de TBP bacilifera encontrada fue del 0,69\% $(n=1)$. Los $S R$ representaron el 14,7\% (n=21) de la muestra y estuvieron asociados al grupo de pacientes ingresados hacía más de dos años y sin perspectiva de alta hospitalaria $(p=0,01)$ y el hábito de fumar $(p=0,02)$. Conclusión: En este grupo se encontró elevada prevalencia de TBP bacilifera al comparar a la población brasileña y los SR estuvieron asociados al mayor tiempo de ingreso hospitalario $y$ el habito de fumar.

Descriptores: Tuberculosis Pulmonar; Hospitales Psiquiátricos; Hábito de Fumar.

\section{INTRODUÇÃO}

A tuberculose (TB) é uma doença infecciosa crônica causada pelo Mycobacterium tuberculosis (Mtb), cuja transmissão ocorre predominantemente por via aérea. Isso justifica o fato de $80 \%$ dos infectados apresentarem a tuberculose pulmonar (TBP), de maior relevância clínicoepidemiológica $^{(1-3)}$.

Relevante problema de saúde pública, a TB é a segunda doença infecciosa que mais mata no mundo ${ }^{(4-9)}$. Tanto a intensificação dos fluxos migratórios quanto a pandemia da Síndrome de Imunodeficiência Adquirida (AIDS) possuem papéis importantes na manutenção das incidências de TB em todo o planeta, uma vez que indivíduos soropositivos são dez vezes mais susceptíveis ao Mtb; dessa forma, representam elevado risco de transmissão a contatos ${ }^{(3-5,10-12)}$.

De acordo com a Organização Mundial da Saúde (OMS) e outros autores, aproximadamente dois bilhões de pessoas vivem com a forma latente da infecção pelo Mtb, e cerca de 7 milhões sofrem de formas ativas da doença no mundo. Oito milhões e seiscentas mil pessoas adoeceram por TB em 2012, resultando em 1,3 milhão de mortes, particularmente em países subdesenvolvidos ou em desenvolvimento ${ }^{(2,6,13-15)}$. Estima-se prevalência de 110.000 infectados no Brasil, com aproximadamente 70.000 casos novos e 5.000 mortes contabilizadas a cada ano. Tal fato classifica o país em $17^{\circ}$ lugar entre os 22 com maior carga de TB (do inglês "high TB burden countries"), responsáveis por $80 \%$ de todos os casos detectados mundialmente ${ }^{(15)}$.

A prevalência de TB entre sujeitos internados ou que trabalham em hospitais psiquiátricos, sistemas carcerários ou asilos é cerca de 16 vezes superior àquela na população geral, o que representa alto risco de surtos e disseminação de cepas multirresistentes ${ }^{(16)}$. Embora a TB seja considerada grave problema de saúde pública, a revisão de literatura realizada durante a elaboração desta pesquisa não encontrou estudo de busca ativa conduzido em instituições psiquiátricas do estado de Alagoas antes desta iniciativa. Apenas um trabalho realizado em ambiente hospitalar psiquiátrico foi encontrado no âmbito nacional.

À vista do exposto, o presente trabalho objetiva avaliar a prevalência de tuberculose pulmonar (TBP) bacilífera e sintomáticos respiratórios (SR) em um hospital psiquiátrico público de referência em Alagoas.

\section{MÉTODOS}

Estudo transversal de abordagem quantitativa, conduzido no Hospital Escola Portugal Ramalho(HEPR), em Alagoas, por meio da busca ativa por pacientes sintomáticos respiratórios e casos de TBP bacilífera, de março a abril de 2013. O número total de leitos disponíveis na instituição é de 160 , sendo 95 na ala masculina, a qual contém uma unidade para usuários de drogas com 27 leitos, e 65 femininos. No período de realização da pesquisa, 146 leitos estavam ocupados, incluindo 18 "residentes" (internados na instituição há mais de dois anos e sem perspectiva de alta devido à falta de suporte social ou referência familiar). Um paciente recusou participação e dois foram excluídos por insuficiência de dados, estabelecendo-se, assim, uma amostra fixa composta por 143 internos.

Os indivíduos internados no HEPR foram incluídos na pesquisa apenas após registro da assinatura de um tutor legal - familiar ou o diretor do hospital - em Termos de Consentimento Livre e Esclarecido (TCLEs). Os critérios de exclusão foram a retirada de consentimento e a insuficiência de dados.

Um questionário específico foi elaborado para coleta de dados. Baseados na revisão de prontuários e em exames clínicos dos internos, questionários contendo as variáveis "idade", "tempo de internação", "sexo", "cor da pele", "nível educacional", "condição habitacional prévia" (condição anterior à internação, exceto em caso de paciente residente - ver definição adiante), "número de contatos antes da internação", "condições debilitantes" (HIV, alcoolismo, hábito de fumar, uso de drogas ilícitas, doenças infecciosas), "sintomas respiratórios" (presença de algum dos listados/ nenhum), "TB atual" (sim/não) e "medicamento(s) em uso" 
foram preenchidos. Pacientes internados na instituição por no mínimo dois anos, sem perspectiva de alta devido à falta de suporte social ou referência familiar, são classificados como residentes. Dois grupos foram então formados: SR (contendo pacientes sintomáticos respiratórios) e AR (contendo pacientes assintomáticos) ${ }^{(17,18)}$.

Os critérios de inclusão considerados para composição de SR (sintomas clássicos de TBP) - tosse por duas ou mais semanas, dispneia ou dor torácica (acompanhada ou não por expectoração, hemoptise ou sibilância) - foram baseados na Abordagem Prática à Saúde Pulmonar (do inglês: Practical Approach to Lung Health - PAL), promovida pela Organização Mundial da Saúde, e nas III Diretrizes para Tuberculose da Sociedade Brasileira de Pneumologia e Tisiologia $(\mathrm{SBPT})^{(8,14)}$. Os internos pertencentes à SR foram submetidos à coleta de escarro. Duas amostras de material biológico por paciente (a segunda sempre em jejum) foram coletadas em um intervalo de 24 a 48 horas, em espaço aberto e ventilado, e armazenadas em recipientes refrigerados, cuja temperatura interna foi mantida a $+4^{\circ} \mathrm{C}$ até a análise executada no Laboratório Central de Saúde Pública de Alagoas (LACEN/AL). No laboratório, baciloscopias foram realizadas na busca por bacilos álcoolácido resistentes (BAARs), utilizando a coloração de ZiehlNeelsen ${ }^{(8,18)}$. Nesta pesquisa, a confirmação de casos de TBP foi realizada por meio de duas baciloscopias BAARpositivas, seguindo as recomendações da Organização PanAmericana de Saúde (OPAS) e da SBPT, as quais incluem o incentivo à detecção precoce de indivíduos bacilíferos ${ }^{(8,13)}$.

Os softwares SPSS, Statistics 18 e EpiInfo ${ }^{\circledR} 3.4 .2$ foram utilizados nas análises estatísticas. Os testes Qui-quadrado de Yates ou Pearson e Razão de Prevalência (RP) foram usados na comparação entre grupos quanto aos fatores de risco apresentados. Um valor " $\mathrm{p}<0.05$ " foi considerado como significância estatística.

A pesquisa segue os preceitos da Declaração de Helsinki e da Resolução n 466, de 12 de dezembro de 2012, do Conselho Nacional de Saúde ${ }^{(19)}$. O estudo foi submetido ao Comitê de Ética em Pesquisa Local e aprovado por meio do parecer $\mathrm{n}^{\circ} 1543 / 12$, emitido em janeiro de $2013^{(20,21)}$.

\section{RESULTADOS}

A maioria dos 143 participantes pertencia ao sexo masculino $(61,5 \%, n=88)$, dos quais $51,7 \%(n=74)$ eram adultos jovens (20-39 anos de idade) e 79,7\% ( $n=114)$, pardos. A média de idade encontrada foi de $36,4(\mathrm{DP}=$ $\pm 14.2)$ anos.

Com relação às condições habitacionais prévias, 70,6\% $(n=101)$ dos internos moravam em casas de alvenaria antes da internação, 12,6\% $(\mathrm{n}=18)$ eram pacientes residentes, $9,1 \%(\mathrm{n}=13)$ viviam em moradias precárias ou não possuíam casa própria (sete viviam nas ruas, cinco em casas de taipa e um em barraco de lona) e 7\% ( $\mathrm{n}=10)$ não possuíam qualquer informação descrita no campo.

No que diz respeito ao nível educacional, foi observada a predominância de baixo nível educacional, uma vez que $46,8 \%(n=67)$ dos pacientes pesquisados não haviam completado o ensino fundamental e $30,8 \%(n=44)$ eram analfabetos. Apenas 7,7\% (n=11) dos entrevistados concluíram todo ou em parte o ensino médio (Tabela I).

Já em termos de condições debilitantes associadas, identificou-se alta ocorrência de fumantes, $23,8 \%(n=34)$ da amostra. Além disso, casos de alcoolismo - 20,3\% ( $\mathrm{n}=29)$,

Tabela I - Perfil sociodemográfico dos pacientes internados no Hospital Escola Portugal Ramalho (HEPR). Alagoas, 2013.

\begin{tabular}{lccc}
\hline Variáveis & Total $-\mathbf{n} \mathbf{( \% )}$ & SR $-\mathbf{n ~ ( \% )}$ & AR - n (\%) \\
\hline Sexo & $55(38,5)$ & $9(16,4)$ & $46(83,6)$ \\
Feminino & $88(61,5)$ & $12(13,6)$ & $76(86,4)$ \\
Masculino & & & \\
Cor da pele & $16(11,2)$ & $4(25)$ & $12(75)$ \\
Branca & $13(9,1)$ & $2(15,38)$ & $11(84,62)$ \\
Negra & $114(79,7)$ & $15(13,15)$ & $99(86,85)$ \\
Parda & & & \\
Faixa etária & $15(10,5)$ & $2(13,33)$ & $13(86,67)$ \\
$10-19$ anos & $74(51,7)$ & $7(9,46)$ & $67(90,54)$ \\
$20-39$ anos & $40(28,0)$ & $10(25)$ & $30(75)$ \\
$40-59$ anos & $13(9,1)$ & $2(15,38)$ & $11(84,62)$ \\
$\geq 60$ anos & $1(0,7)$ & - & $1(100)$ \\
Ignorado & & & \\
\hline
\end{tabular}

$\mathrm{SR}=$ sintomáticos respiratórios; $\mathrm{AR}=$ assintomáticos. 
Tabela II - Distribuição das condições debilitantes encontradas nos pacientes dos grupos "sintomas respiratórios" (SR) e “assintomáticos” (AR), atendidos no Hospital Escola Portugal Ramalho (HEPR). Alagoas, 2013.

\begin{tabular}{|c|c|c|c|c|c|c|}
\hline \multirow{2}{*}{ Condições } & \multicolumn{2}{|c|}{ Casos SR } & \multicolumn{2}{|c|}{ Casos AR } & \multirow{2}{*}{$\mathbf{R P}$} & \multirow{2}{*}{ Teste $\chi^{2}$} \\
\hline & $\mathbf{n}$ & $\%$ & n & $\%$ & & \\
\hline \multicolumn{7}{|l|}{ Alcoolismo } \\
\hline Sim & 2 & 6,9 & 27 & 93,1 & 0,37 & $1,76(p=0,18)$ \\
\hline Não & 19 & 16,6 & 95 & 83,4 & & \\
\hline \multicolumn{7}{|c|}{ Hábito de fumar } \\
\hline Sim & 9 & 26,5 & 25 & 73,5 & 2,91 & $4,945(\mathrm{p}=0,02)$ \\
\hline Não & 12 & 11,0 & 97 & 89,0 & & \\
\hline \multicolumn{7}{|c|}{ Uso de drogas ilícitas } \\
\hline Sim & 4 & 19,0 & 17 & 81,0 & 1,62 & $0,37(p=0,54)$ \\
\hline Não & 17 & 14,0 & 105 & 86,0 & & \\
\hline \multicolumn{7}{|l|}{ SIDA/AIDS } \\
\hline Sim & 1 & 50,0 & 1 & 50,0 & 6,05 & $2,02(p=0,15)$ \\
\hline Não & 20 & 14,2 & 121 & 85,8 & & \\
\hline \multicolumn{7}{|l|}{ Sífilis** } \\
\hline Sim & 1 & 100,0 & 0 & 0 & inválida & ---------------- \\
\hline Não & 20 & 14,1 & 122 & 85,9 & & \\
\hline
\end{tabular}

$\mathrm{SR}=$ sintomáticos respiratórios; $\mathrm{AR}=$ assintomáticos. *RP: razão de prevalência.**Teste qui-quadrado de Pearson: 5,85 ( $\mathrm{p}<0.05$ ), no entanto, é invalido porque duas células (50\%) apresentam valor esperado inferior a 5.

dependência de drogas ilícitas $-14,7 \%(\mathrm{n}=21)$, positividade sorológica para o vírus HIV - 1,4\% ( $\mathrm{n}=2)$ e sífilis $0,7 \%(\mathrm{n}=1)$ - foram encontrados. Constatou-se, ainda, a concorrência dessas condições ( 2 a 3, simultaneamente) em, respectivamente, $9,8 \%(\mathrm{n}=14)$ e $4,9 \%(\mathrm{n}=7)$ dos participantes (Tabela II).

Vinte e um pacientes $(14,7 \%)$ - 9 mulheres e 12 homens - compuseram o grupo SR, cujas médias de idade e tempo de internação foram, respectivamente, 41,8 anos e 1.456 dias (cerca de 48 meses e meio). Indivíduos do grupo AR $(85,3 \%, n=122)$ foram internados, em média, por 357,5 dias (aproximadamente 12 meses). Os residentes, por sua vez, apresentaram tempo médio de internação de 4.475 dias (cerca de 12 anos e 3 meses).

A comparação entre os grupos SR e AR revelou associação entre o hábito de fumar e o grupo $\mathrm{SR}(\mathrm{RP}=2,91$; p=0,026) (Tabela II). Foi ainda detectada associação estatisticamente significativa entre boa condição habitacional (casa de alvenaria) e AR ( $\mathrm{RP}=3,719 ; \mathrm{p}=0,013)$ (Tabela III). Por outro lado, foi observado que os residentes eram significativamente mais sintomáticos que os não residentes ( $R P=4,59 ; p=0,01)$ (Tabela IV).

A prevalência de TBP bacilífera encontrada no grupo avaliado foi de $0,69 \%(n=1)$. O indivíduo doente, internado na instituição pela terceira vez devido à exacerbação de seu transtorno mental (esquizofrenia paranoide), foi diagnosticado por meio de baciloscopias positivas obtidas 53 dias após a última admissão hospitalar. $\mathrm{O}$ paciente estava no quarto mês de tratamento (fase de manutenção) à época da execução deste trabalho ${ }^{(8)} \mathrm{e}$ recebeu alta no dia 9 de abril de 2013, após melhora dos sintomas psicóticos e por solicitação da irmã, o que ocorreu, contudo, antes da finalização do tratamento para TBP. 
Tabela III - Quadro comparativo da condição habitacional prévia dos pacientes nos grupos "sintomas respiratórios” (SR) e "assintomáticos" (AR), atendidos no Hospital Escola Portugal Ramalho (HEPR). Alagoas, 2013.

\begin{tabular}{|c|c|c|c|c|c|c|}
\hline \multirow{3}{*}{ Vivia em casa de alvenaria } & \multicolumn{4}{|c|}{ Grupos } & \multirow{3}{*}{$\mathbf{R P}$} & \multirow{3}{*}{$\begin{array}{l}\text { Teste } \chi^{2} \\
\text { (Yates) }\end{array}$} \\
\hline & \multicolumn{2}{|c|}{$\mathbf{A R}$} & \multicolumn{2}{|c|}{ SR } & & \\
\hline & $\mathrm{n}$ & $\%$ & $\mathrm{n}$ & $\%$ & & \\
\hline Sim & 90 & 89,1 & 11 & 10,9 & 3,719 & 6,121 \\
\hline Não & 22 & 68,7 & 10 & 31,3 & & $(p=0,013)$ \\
\hline Total* & 112 & 84,2 & 21 & 15,8 & & \\
\hline
\end{tabular}

$\mathrm{SR}=$ sintomáticos respiratórios; $\mathrm{AR}=$ assintomáticos. * RP: razão de prevalência. *133 é o total após a remoção dos campos ignorado/ branco.

Tabela IV - Distribuição de pacientes residentes no Hospital Escola Portugal Ramalho (HEPR) entre os grupos "sintomas respiratórios" (SR) e "assintomáticos" (AR), atendidos. Alagoas, 2013.

\begin{tabular}{|c|c|c|c|c|c|c|}
\hline \multirow{3}{*}{ Residente* no HEPR } & \multicolumn{4}{|c|}{ Grupos } & \multirow{3}{*}{$\mathbf{R P}$} & \multirow{3}{*}{$\begin{array}{l}\text { Teste } \chi^{2} \\
\text { (Yates) }\end{array}$} \\
\hline & \multicolumn{2}{|c|}{ SR } & \multicolumn{2}{|c|}{$\mathbf{A R}$} & & \\
\hline & n & $\%$ & $n$ & $\%$ & & \\
\hline Sim & 7 & 38,9 & 11 & 61,1 & 4,590 & 6,465 \\
\hline Não & 14 & 12,2 & 101 & 87,8 & & $(\mathrm{p}=0,01)$ \\
\hline Total** & 21 & 15,8 & 112 & 84,2 & & \\
\hline
\end{tabular}

HEPR=Hospital Escola Portugal Ramalho; $\mathrm{SR}=$ sintomáticos respiratórios; $\mathrm{AR}=$ assintomáticos. *RP: razão de prevalência. *Pacientes internados na instituição por no mínimo dois anos, sem perspectiva de alta devido à falta de suporte social e referência familiar. **133 é o total após a remoção dos campos ignorado/branco.

\section{DISCUSSÃO}

A prevalência de TBP bacilífera no hospital psiquiátrico de referência em Alagoas é mais de 12 vezes maior que a prevalência de TB - todas as formas - estimada no país. Esse achado corrobora resultados de outros estudos, os quais apontam prevalência mais elevada da doença em cenários de privação da liberdade, quando comparada àquela na população geral ${ }^{(16)}$. $\mathrm{O}$ valor encontrado é quase 33 vezes maior que as taxas de incidência média de TBP bacilífera no Brasil (21,37/100.000) e na região Nordeste (21,27/100.000), no período de 2009 a 2012, segundo dados tabulados a partir do Sistema de Informação de Agravos de Notificação (SINAN). Em Alagoas, no mesmo período, 2.420 novos casos de tuberculose pulmonar bacilífera foram contabilizados $-63,2 \%$ em homens, a maioria na faixa etária de 20-39 anos -, com taxas decrescentes durante o período $(19,61 ; 19,52 ; 18,96 ; 18,83 / 100.000$, de 2009 a 2012, respectivamente) ${ }^{(13,22)}$. A ocorrência descrita neste trabalho é ligeiramente superior ( 36,3 vezes) à incidência média de TBP bacilífera em Alagoas (19,23/100.000), no entanto, pode ser hiperestimada, por possível subnotificação no estado ${ }^{(22)}$. O resultado exposto é muito preocupante, principalmente se levado em consideração o fato de que o acompanhamento da população de internos foi breve. Chama-se atenção para o valor epidemiológico desse tipo de ambiente de alto risco, que pode ser fonte de perpetuação da tuberculose na comunidade ${ }^{(17,23)}$.

No tocante ao caso de TBP detectado na amostra, este trabalho condiz com a literatura nacional em relação a gênero e idade ${ }^{(8)}$. Já no que diz respeito ao nível de escolaridade, o resultado aqui exposto diverge do perfil nacional, uma vez que o indivíduo acometido pela doença concluíra o ensino médio, o que não ocorre na maioria dos casos (analfabetos ou com fundamental incompleto).

Embora já estivesse no $4^{\circ}$ mês do esquema terapêutico, o paciente continuava a apresentar tosse ocasional. Devido a isso, foi incluído no grupo SR, a fim de se identificar possível 
falha no tratamento. Apesar de os laudos das baciloscopias realizadas durante este estudo terem resultados negativos, vários fatores de risco, como alcoolismo, solidão (falta de supervisão), comprometimento cognitivo e relatos prévios de rejeição à medicação, desnutrição, limitação das medidas preventivas/terapêuticas e falta de higiene, são fortemente favoráveis à interrupção do tratamento e recaída ${ }^{(24,25)}$.

O confinamento prolongado no hospital psiquiátrico pode representar maior risco de transmissão diante de casos confirmados que porventura sofram falha terapêutica, ou de casos surgidos a partir da ativação de infecção latente, após exposição a fatores de risco relacionados ao ambiente, como superlotação, condições precárias de higiene e ventilação ${ }^{(16,18,26)}$. A associação descrita entre residentes - internados em média há cerca de 12 anos e 3 meses e o grupo SR reforça o fato de que há risco atribuído ao ambiente de confinamento coletivo, e ele é potencializado por longas permanências ${ }^{(16,18,26)}$.

Em 2002, pesquisadores brasileiros realizaram um estudo transversal em um hospital psiquiátrico de Goiás que revelou maior taxa de positividade da prova tuberculínica (PT) entre profissionais de saúde que trabalharam no local por maior período de tempo (30 meses e 18 meses, respectivamente, para os grupos de PT positiva e negativa) (16).

A alta ocorrência de condições debilitantes - tabagismo (23,8\%), alcoolismo (20,3\%), dependência de drogas ilícitas $(14,7 \%)$ e AIDS $(1,4 \%)$ - aumentaria a susceptibilidade desses indivíduos, o que parece ser consequência de efeito imunossupressor, decorrente diretamente da exposição crônica a essas substâncias e condições, ou devido à desnutrição relacionada a comportamento alterado, resultante de desordem mental ou síndrome de abstinência $^{(17)}$.

Das condições descritas, o presente trabalho mostrou associação estatisticamente significativa apenas entre o hábito de fumar e o grupo SR. De fato, o tabagismo é fator de risco muito bem conhecido para a TBP e está relacionado à maior infectividade, morbidade, mortalidade e taxas de recidiva em fumantes ativos e passivos, por isso, é alvo de estratégias anti- $\mathrm{TB}^{(8,27)}$.

Este trabalho mostrou, ainda, associação estatisticamente significativa entre boa condição habitacional (casa de alvenaria) e o grupo AR. A partir daí, infere-se que a presença de contextos socioeconômicos desfavoráveis aumenta o risco do surgimento de sintomas respiratórios, conforme diversos estudos ${ }^{(4,5,11,27)}$. Tal associação sugere vulnerabilidade a infecções respiratórias, incluindo TBP.

A baciloscopia foi o teste diagnóstico de escolha deste trabalho, por ser não invasivo, seguro, rápido e barato.
Além disso, o principal objetivo dos autores foi identificar pacientes bacilíferos, os quais respondem por $60-80 \%$ dos casos de TBP e são diagnosticados pelo teste ${ }^{(25)}$. Casos não bacilíferos representam baixo risco de transmissão, portanto, é improvável que iniciem surto. Tanto a OPAS quanto a SBPT recomendam condução de busca ativa de casos de TB ativa em serviços de saúde ou comunidades de regiões com alta prevalência da doença, contribuindo para detecção precoce de casos bacilíferos, o que diminui o tempo de exposição da comunidade. Tal estratégia consiste na realização de baciloscopias após a identificação de indivíduos sintomáticos respiratórios ${ }^{(8,25,28,29)}$.

É crucial registrar as limitações na realização deste estudo. Os pesquisadores lidaram com pacientes cujas capacidades de julgamento e cognição são frequentemente comprometidas, o que dificultou algumas coletas de escarro. Além disso, apenas metade dos pacientes com TBP é positiva à baciloscopia, e até $30 \%$ sequer apresenta expectoração espontânea durante a fase inicial da doença, o que pode ter contribuído com alguns resultados falsonegativos $^{(17)}$.

A busca ativa por contatos de enfermos com TB, por meio de PT, radiografia de tórax e baciloscopia, é formalmente recomendada pelo Ministério da Saúde do Brasil e pela SBPT, a fim de avaliar se se deve tratar eventual tuberculose latente entre os contatos ${ }^{(8)}$. Tal conduta não foi necessária nesta pesquisa, já que o único caso identificado vivia sozinho antes da internação e manteve-se em isolamento durante os primeiros 30 dias de tratamento.

\section{CONCLUSÃO}

A prevalência de TBP bacilífera encontrada no HEPR foi 33 vezes superior àquela na população brasileira e 12 vezes superior à prevalência de todas as formas de TB no país. Dessa forma, ambientes de risco como o abordado pelo presente trabalho revestem-se de extrema importância epidemiológica e devem ser alvos estratégicos no controle da doença na comunidade. A pesquisa também destaca que a carga de TB continua a ser significativa em Alagoas e no país. Uma pesquisa adicional é necessária não apenas para melhor compreender o comportamento da doença em cenários semelhantes, mas também para apontar soluções que fundamentem estratégias de saúde pública para enfrentar esse grave problema.

\section{AGRADECIMENTOS}

Os autores são gratos aos funcionários do Hospital Escola Portugal Ramalho pela sua colaboração, à equipe do Laboratório Central de Saúde Pública de Alagoas (LACEN/ $\mathrm{AL}$ ) pelos auxílios técnico e material (análise bacteriológica 
e kits de coleta, respectivamente) e à pesquisadora June Penman, $\mathrm{PhD}$, pela revisão deste trabalho.

\section{CONFLITOS DE INTERESSE}

Os autores deste trabalho não possuem conflitos de interesse de qualquer natureza a declarar.

\section{REFERÊNCIAS}

1. Exel AL, Torres RM, Ferro BMS, Silva MAS, Amorim RC. Perfil dos indivíduos com diagnóstico de tuberculose, notificados no Hospital Escola Dr. Hélvio Auto, Estado de Alagoas, Brasil. Alagoas: Secretaria de Estado da Saúde de Alagoas; 2009 [acesso em 2015 Maio 19]. Disponível em: http://www.siteantigo. saude.al.gov.br:82/artigocientifico/documentos/ perfildosindividuoscom

2. Walzl G, Ronacher K, Hanekom W, Scriba TJ, Zumla A. Immunological biomarkers of tuberculosis. Nat Rev Immunol. 2011;11:343-54.

3. Silva JRL, Boéchat N. O ressurgimento da tuberculose e o impacto do estudo da imunopatogenia pulmonar. J Bras Pneumol. 2004;30(4):478-84.

4. Alemie GA, Gebreselassie F. Common types of tuberculosis and co-infection with HIV at private health institutions in Ethiopia: a cross sectional study. BMC Public Health. 2014;14:319.

5. Ibrahim LM, Hadejia IS, Nguku P, Dankoli R, Waziri NE, Akhimien MO, et al. Factors associated with interruption of treatment among pulmonary tuberculosis patients in Plateau State, Nigeria, 2011. Pan Afr Med J. 2014;17:78.

6. Mandal P, Craxton R, Chalmers JD, Gilhooley S, Laurenson IF, McSparron $\mathrm{C}$ et al. Contact tracing in pulmonary and non-pulmonary tuberculosis. QJM. 2012;105(8):741-7.

7. Qiu Z, Zhang M, Zhu Y, Zheng F, Lu P, Liu H et al. Multifunctional CD4 cell responses in patients with active tuberculosis. Sci Rep. 2012;2:216.

8. BTA Committee on Tuberculosis, BTA Guidelines on Tuberculosis Work Group. III Diretrizes para Tuberculose da Sociedade Brasileira de Pneumologia e Tisiologia (SBPT). J Bras Pneumol. 2009;35(10):101848.

9. Hijar MA, Procopio MJ, De Freitas LSR, Guedes R, Bethlem EP. Epidemiology of tuberculosis in the world, Brazil and Rio de Janeiro. Pulmão RJ. 2005;14(4):3104.

Rev Bras Promoç Saúde, Fortaleza, 28(4): 521-528, out./dez., 2015
10. Bhat J, Rao VG, Gopi PG, Yadav R, Selvakumar N, Tiwari B et al. Prevalence of pulmonary tuberculosis amongst the tribal population of Madhya Pradesh, Central India. Int J Epidemiol. 2009;38(4):1026-32.

11. Nájera-Ortiz JC, Sánchez-Pérez HJ, Ochoa-Días H, Arana-Cedeño M, Lezama MAS, Mateo MM. Demographic, health services and socio-economic factors associated with pulmonary tuberculosis mortality in Los Altos Region of Chiapas, Mexico. Int J Epidemiol. 2008;37(4):786-95.

12. Wu P, Lau EHY, Cowling BJ, Leung CC, Tam CM, Leung GM. The transmission dynamics of tuberculosis in a recently developed chinese city. Plos One. 2010;5(5):e10468.

13. Ministério da Saúde (BR). Plano Estratégico para o Controle da Tuberculose, Brasil 2007-2015. Brasília: Ministério da Saúde; 2006.

14. Organização Mundial da Saúde. Global tuberculosis control epidemiology, strategy, financing. Genebra: OMS; 2008.

15. Organização Mundial da Saúde. Global tuberculosis report. 2013. Genebra: OMS; 2013.

16. Costa HCG, Malaspina AC, Mello FAF, Leite CQF. Ocorrência de tuberculose em um hospital psiquiátrico do interior de Goiás. J Bras Pneumol. 2006;32(6):56672.

17. Capuano DA, Ward JK, Fortes CQ, Sá FC. Busca ativa de casos de tuberculose pulmonar em uma unidade de atendimento em farmacodependência no Município de São Paulo (agosto de 1999 a agosto de 2000). Rev Bras Epidemiol. 2003;6(3):255-61.

18. Vieira AA, Ribeiro SA, Siqueira AM, Galesi VMN, Golub JE et al. Prevalence of patients with respiratory symptoms through active case finding and diagnosis of pulmonary tuberculosis among prisoners and related predictors in a jail in the city of Carapicuíba, Brazil. Rev Bras Epidemiol. 2010;13(4):641-50.

19. Ministério da Saúde (BR). Resolução n 466, de 12 de dezembro de 2012. Aprova as diretrizes e normas regulamentadoras de pesquisas envolvendo seres humanos [estatuto na internet]. Diário Oficial da República Federativa do Brasil, Brasília (DF), 2012 dez 12; Seção 1:137.

20. Universidade Federal de Alagoas. Informação sobre o Comitê de Ética em Pesquisa (CEP). Maceió: Universidade Federal de Alagoas; 2012 [acesso em 2012 Out 20]. Disponível em: http://www.ufal.edu.br/ comite-de-etica-em-pesquisa/ 
21. Centro Universitário Cesmac. Informação sobre o Comitê de Ética em Pesquisa (CEP). Maceió: Cesmac; 2012 [acesso em 2012 Out 20]. Disponível em: http:// www.fejal.com.br/cep/

22. Ministério da Saúde (BR). Casos de tuberculose confirmados de 2009 a 2012. Brasília: SinanNetDatasus; 2013 [acesso em 2013 Mar 8]. Disponível em: http://www.tabnet.datasus.gov.br/cgi/idb2012/matriz. htm\#morb

23. Mascarenhas MDM, Araújo LM, Gomes KRO. Perfil epidemiológico da tuberculose entre casos notificados no Município de Piripiri, Estado do Piauí, Brasil. Epidemiol Serv Saúde. 2005;14(1):7-14.

24. Campani STA, Moreira JS, Tietbohel CN. Fatores preditores para o abandono do tratamento da tuberculose pulmonar preconizado pelo Ministério da Saúde do Brasil na cidade de Porto Alegre (RS). Rev Bras Epidemiol. 2011;37(6):776-82.

25. Ministério da Saúde (BR). Manual de recomendações para o controle da tuberculose no Brasil. Brasília: Ministério da Saúde; 2011.

26. Nogueira PA, Abrahão RMCM, Galesi VMN. Infecção tuberculosa latente em profissionais contatos e não contatos de detentos de duas penitenciárias do Estado de São Paulo, Brasil, 2008. Rev Bras Epidemiol. 2011;14(3):486-94.
27. Ferreira AAA, Queiroz KCS, Torres KP, Ferreira MAF, Accioly H, Alves MSCF. Os fatores associados à tuberculose pulmonar e a baciloscopia: uma contribuição ao diagnóstico nos serviços de saúde pública. Rev Bras Epidemiol. 2005;(8)2:142-9.

28. Organização Panamericana de Saúde. Regional strategy for the control of tuberculosis for 2005-2015. Washington: OMS; 2005.

29. Freitas FTM, Yokota RTC, Castro APB, Andrade SSCA, Nascimento GL, Moura NFO et al. Prevalência de sintomáticos respiratórios em regiões do Distrito Federal, Brasil. Rev Panam Salud Pública. 2011;29(6):451-6.

\section{Endereço para correspondência:}

George Vasconcelos Calheiros de Oliveira Costa

Av Lourival Melo Mota, S/N

Bairro: Tabuleiro dos Martins

CEP: 57072-900 - Maceió - AL - Brasil

E-mail: george_vcoc@hotmail.com 